REVUE HYBRIDE DE L'ÉDUCATION

\title{
Éditorial \\ Créer la synergie entre les milieux de pratique et de recherche
}

Marie-Pierre Baron

Directrice de la RHÉ, professeure à l'Université du Québec à Chicoutimi 


\section{REVUE HYBRIDE DE L'ÉDUCATION}

\section{Avant propos}

Les chercheurs en éducation se penchent sur les enjeux et les problématiques vécus dans les milieux de pratique, qu'ils soient, par exemple, pédagogiques, didactiques, psychopédagogiques ou sociaux. Cependant, l'enjeu principal du chercheur (si on exclut l'accès au financement!) réside dans les retombées de ses travaux. Comment s'assurer que les chercheurs répondent réellement aux besoins des praticiens et même que les questions traitées soient le reflet de ce qui est vécu dans les milieux de pratique? Comment faire en sorte que les travaux de recherche soient discutés dans les milieux d'enseignement et vice versa? Le conseil supérieur de l'éducation (2016) souhaite également que le développement professionnel continu des enseignants en exercice se base sur les connaissances issues de la recherche en éducation et que les acteurs des milieux de pratique diffusent et partagent leurs expériences positives et leurs expertises singulières. Peu importe le statut d'emploi, nous visons l'atteinte du même objectif: créer des ponts entre les différents milieux de l'éducation.

C'est ce que la ligne éditoriale de la RHÉ propose : établir un lieu de rencontre pour faire valoir les différents possibles de la collaboration entre la recherche et la pratique. Le but est de créer une réelle synergie entre les chercheurs universitaires et les partenaires des milieux scolaires. C'est d'ailleurs ce dont a explicitement traité le premier numéro thématique de la RHÉ en présentant différents exemples de transformation des pratiques en collaboration avec les milieux scolaires du Saguenay-Lac-Saint-Jean. En donnant la parole à tous, et ce, par l'intermédiaire des différentes rubriques professionnelles et scientifiques, l'équipe éditoriale de la RHÉ fait le pari du libre accès et de la démocratisation de l'écrit pour que tous puissent contribuer à la transformation des pratiques en éducation. 


\section{REVUE HYBRIDE DE L'ÉDUCATION}

Dans le numéro du printemps 2018, nous laissons la place aux praticiens, à la relève scientifique et aux chercheurs. Ces articles pour lesquels la collaboration s'est déroulée, tant dans la réalisation de projets que dans l'écriture, font foi des efforts mis en place pour décloisonner les milieux de l'éducation et faire valoir leur interdépendance.

\section{Aperçu des contributions}

Les intentions du comité scientifique sont nobles : donner la parole à tous. Cependant, la réalité des milieux de pratique peut rendre difficile la publication d'articles professionnels, notamment en raison de la complexité de leur tâche et du temps nécessaire à l'écriture. C'est pour cette raison qu'en plus de publier avec des chercheurs les projets réalisés dans les classes, ce numéro propose également des articles issus d'entrevues réalisées par des étudiants de premier et de troisième cycle qui se sont rendus dans les classes dans le but de s'entretenir avec des praticiens. À l'image de la RHÉ, des articles ont donc été écrits par la relève scientifique en collaboration avec les enseignants et des chercheurs universitaires.

Le premier article de Gagné, Boily et Coulombe traite également de l'insertion socioprofessionnelle chez les étudiants adultes. L'article porte de l'implication au sein d'un service de cantine étudiante au sein d'un centre de formation de la Commission scolaire du Lac-Saint-Jean.

Le second article de Gagné, Cloutier, Duchesne et Girard présente une initiative pédagogique de la Commission scolaire des Rives-du-Saguenay. II discute de l'utilisation du carnet d'employabilité à la formation professionnelle dans le but de favoriser l'insertion socioprofessionnelle des finissants.

Le troisième article de Gignac, Normandeau et Monney porte sur le développement de l'autonomie et du gout d'apprendre par l'utilisation du plan de travail, dans une classe du Saguenay-Lac-Saint-Jean. II est issu 


\section{REVUE HYBRIDE DE L'ÉDUCATION}

d'une entrevue réalisée auprès d'une enseignante d'une école trilingue de Saguenay.

Le quatrième article est un article régulier de la rubrique Milieu de pratique, plus précisément un article professionnel présentant les fruits d'une collaboration entre des enseignantes et un didacticien des mathématiques. Raymond, Barry et Plourde y exposent leurs réflexions pédagogiques et didactiques en ce qui a trait à l'utilisation du plan de travail au primaire comme un organisateur réfléchi et efficace.

Le numéro se conclut par un article de la rubrique Place à la relève scientifique. Turcotte est étudiant à la maitrise en éducation tandis que Doucet et Baron sont professeures-chercheuses. Ils proposent un article réflexif portant sur l'intérêt de s'intéresser aux stratégies d'études des étudiants du collégial ayant un trouble d'apprentissage ou un trouble déficitaire de l'attention avec ou sans hyperactivité.

\section{Remerciements}

La publication de ce premier numéro régulier de la Revue hybride de l'éducation a été possible grâce au travail de personnes impliquées et dévouées. Entre la publication du numéro de lancement et de celui-ci, plusieurs changements sont survenus, plusieurs ajustements ont été faits, dont le changement de plateforme de publication sur OJS-3. Un grand merci aux auteurs et aux évaluateurs des articles. Pour leur regard éclairé, leur disponibilité et leur travail hors pair, merci aux membres du comité scientifique composé de Nicole Monney (UQAC), Souleymane Barry (UQAC) et Jean Bernatchez (UQAR). Merci également à monsieur Ugo Collard-Fortin, doctorant en éducation et coordonnateur de la RHÉ qui nous laisse profiter de ses conseils avisés et de son expertise dans le domaine de la publication. Merci également à Marie-Élaine Gignac pour la révision linguistique et le travail de mise en page. Finalement, merci au 


\section{REVUE HYBRIDE DE L'ÉDUCATION}

Département des sciences de l'éducation et à l'Université du Québec à Chicoutimi, entre autres, pour le support et l'hébergement.

\section{Référence :}

Conseil supérieur de l'éducation. (2016). Mémoire du Conseil supérieur de l'éducation dans le cadre des consultations publiques pour une politique de la réussite éducative. Cinq idées-forces pour soutenir l'élaboration d'une politique de la réussite éducative des jeunes et des adultes. Québec: Conseil supérieur de l'éducation. 